Vol 11, Issue 8, 2018

online - 2455-3891

Print - 0974-2441

$\underline{\text { Research Article }}$

\title{
LETROZOLE WITH OR WITHOUT GONADOTROPIN AS A FIRST-LINE OVULATION INDUCTION IN ANOVULATORY INFERTILE WOMEN DUE TO POLYCYSTIC OVARY SYNDROME
}

\author{
FADIA J ALIZZI* \\ Department of Obstetrics and Gynecology, Al-Mustansiriyah College of Medicine, Baghdad, Iraq. Email: fadiaalizzi46@gmail.com
}

Received: 15 March 2018, Revised and Accepted: 23 April 2018

ABSTRACT

Objectives: The objective is to evaluate the clinical outcome of using letrozole alone or with gonadotropin as first-line ovulation induction in anovulatory infertile polycystic ovary women.

Methods: A prospective single-arm study. 80 infertile polycystic ovarian syndrome (PCOS) women had been recruited between January and October 2017. Letrozole on day 2-3 of the cycle was given. The women are sorted into two groups according to the size of the dominant follicle on day 7 or 8 , Group A (letrozole only group) and Group B (letrozole plus gonadotropin).

Results: In our study, the overall pregnancy rate was (67.5\%) and ovulation rate was 91.3\%. The ovulation rate was significantly higher in Subgroup A than B (97.9\% vs. 81.3\%). Pregnancy rate was higher in Subgroup A (72.9\% vs. 59.4\%), but it was statistically not significant. The number of follicles was significantly higher in Subgroup B. Endometrial thickness, miscarriages, ovarian hyperstimulation syndrome, and multiple pregnancies were not statistically significant. Lower cycle number independently and significantly predict clinical pregnancy, while body mass index has a modest effect.

Conclusions: Letrozole alone or in combination with gonadotropin as a first-line treatment in PCOS may be reasonable since this approach may improve the success rate and minimize the overall costs and risks.

Keywords: Clomiphene citrate, Gonadotropin, Letrozole, Ovulation induction, Polycystic ovarian syndrome.

(C) 2018 The Authors. Published by Innovare Academic Sciences Pvt Ltd. This is an open access article under the CC BY license (http://creativecommons. org/licenses/by/4. 0/) DOI: http://dx.doi.org/10.22159/ajpcr.2018.v11i8.25951

\section{INTRODUCTION}

Polycystic ovarian syndrome (PCOS) prevalence in reproductiveaged women varies from $9 \%$ to $18 \%$ depending on definitions and populations studied. It is the most common endocrine disorder and accounts for $\sim 80 \%$ of women with anovulatory infertility [1]. According to the Rotterdam criteria, diagnosis requires the presence of at least two out of three findings: Hyperandrogenism, ovulatory dysfunction, and polycystic ovaries morphology by ultrasound [2].

Clomiphene citrate (CC), a selective estrogen-receptor modulator, has been used as a first-line ovulation induction treatment in PCOS for decades. Clomiphene has drawbacks, including its overall poor efficacy and an undesirable side-effect profile [3]. Clomiphene resistance, which occurred in $25 \%$ of cases, or clomiphene failure often results in the use of more expensive treatment options for infertility [4]. Although adding metformin may show satisfactory results in women resistant to CC [5], the development of effective, simple, and safe treatments for infertility is an important public health goal [6].

Third-generation aromatase inhibitors (e.g., letrozole) have gained an advantage over other endocrine therapies for the treatment of postmenopausal women in both early and metastatic breast cancer [7].

Over the last years, letrozole, an orally active potent aromatase inhibitor, was widely used for ovulation induction. Numerous original articles, reviews, and meta-analysis have been published. Especially, in women with failure or resistance to CC, letrozole was shown to be very effective both in ovulation rate and live birth rate [8]. Pharmacodynamics of letrozole ensures improved endometrial thickness, cervical mucus, monofollicular, and better folliculogenesis. Therefore, these factors may lead to higher pregnancy rates and a greater likelihood of singleton pregnancy [9].
Gonadotropin preparations, either urinary or recombinant follicle stimulating hormone ( $\mathrm{rFSH}$ ), have been used to stimulate ovulation in women who failed to respond to CC [6]. Gonadotropin may lead to the following drawback effect include cost, the risk of multiple pregnancies, hyperstimulation, and cycle cancellation [10]. rFSH used in combination with CC for CC-resistant PCOS was proven to be more effective in improving the ovulation rate than $\mathrm{rFSH}$ alone [11].

Taking in consideration, the drawback effect of CC versus benefits of letrozole that mentioned above in one hand, and on the other hand, the previous studies which showed that cotreatment with the letrozole significantly reduced the FSH dose required during controlled ovarian stimulation $(\mathrm{COH})$ in women with unexplained infertility $[12,13]$; we proposed the possibility of using it alone or in combination with gonadotropins for ovarian induction for an efficacious approach in women with PCOS.

\section{METHODS}

This study is a prospective single-arm study. It had been approved by an Ethical and Scientific Committee of the Obstetrics and Gynecology department in Al-Yarmouk Teaching Hospital. Once it had been approved, the recruitment started from January 2017 to October 2017.

A total of 80 medically fit infertile women with PCOS had been enrolled in the study after taking informed written consent from all participants and detailed oral and written information concerning the protocol to be used and possible side effect and outcome had been given to them. Modified Rotterdam criteria had been used to diagnose the PCOS. Accordingly, all participating women had ovulatory dysfunction combined with hyperandrogenism and/or ultrasound morphology of polycystic ovary. Thyroid dysfunction, hyperprolactinemia, and other causes of hyperandrogenism had been excluded by 
measurement of serum thyroid-stimulating hormone, prolactin, and 17hydroxyprogesterone.

The entire participant had normal tubal patency test and their partner seminal fluid analysis was normal according to the World Health and obese women for lifestyle modification before and throughout our study.

All the women were asked to come to the infertility clinic on day 2 or 3 of their menstrual cycle wither it was spontaneously descend or induced by using oral progestin, Provera $5 \mathrm{mg}$ /day (medroxyprogesterone acetate ,Pfizer Pharmaceutical ) for 5-10 days.

Baseline transvaginal ultrasound is done, and once no ovarian cyst was seen, oral letrozole $2.5 \mathrm{mg}$ (Femara, Novartis Pharmaceutical) twice daily is given starting from day 2 or day 3 of the cycle for 5 days. The woman is asked to re-come to infertility clinic again at day 7 or day 8 of their cycle for rescanning and according to the size of the dominant follicle at that day the women were divided into two groups. The first group (Group A, letrozole only group) include women who had at least one dominant follicle $10 \mathrm{~mm}$ and above at day 7 or day 8 of the cycle. women who failed to achieve a dominant follicle of $10 \mathrm{~mm}$ and above.

In Group A, the woman will continue to follow-up by transvaginal women will start on day 7 or day 8 of the cycle to use gonadotropin in its recombinant form 75 IU subcutaneously (Gonal, Merck Serono Pharmaceutical) on daily bases for 3 consecutive days (total 3 ampoules) then transvaginal ultrasound repeated again. If mature follicle achieved, no further gonadotropin is given and if not another 1 or 2 doses of gonadotropin (total 5 ampoules) are given subcutaneously for another 2 consecutive days and the women reassessed again. 250 mcg, Merck Serono Pharmaceutical) was administered subcutaneously when one follicle measured $\geq 18 \mathrm{~mm}$ in diameter. HCG injection was concealed if patients have $>3$ follicles (15-18) $\mathrm{mm}$ [14]. In all women, once mature follicle or follicles are achieved (maximum 2 follicles $>16 \mathrm{~mm}$ in Group B and $\geq 17 \mathrm{~mm}$ in Group A), couples were instructed to have regular intercourse 2-3 times a week.

The cycle is repeated for 3 consecutive months in each group unless pregnancy happens. If mature follicles did not achieve by a combination of letrozole and 5 daily doses of gonadotropin for 3 consecutive cycles, we considered it as a failure. 1 week after taking HCG, the woman will be asked to come into the laboratory to have progesterone blood draw to confirm ovulation.

2 weeks from HCG, the woman is asked to return for a blood pregnancy test. Transvaginal ultrasonography was done 4 weeks after positive Organization 2010 cutoff points. We asked and encourage overweight The second groups (Group B, letrozole plus gonadotropin) include ultrasound only till mature follicle achieved, while in Group B, the

Recombinant human chorionic gonadotropin (HCG) alpha (ovidrel,

pregnancy test to confirm the presence of gestational sac with fetal pole and fetal heart pulsation, so the clinical pregnancy was identified.

In the study, the primary outcome was clinical pregnancy while the secondary outcome was ovulation, a number of the dominant follicles, endometrial thickness, and adverse treatment outcome in the form of ovarian hyperstimulation syndrome (OHSS), abortion, and multiple pregnancies.

\section{Statistical analysis}

Discrete variables are described by number and percentage, Chisquare test or Fisher's exact test was used to analyze these variables. Two samples independent t-test used to analyze the differences in means between two groups. Binary logistic regression analysis used to calculate the odds ratio and their 95\% confidence intervals, when the outcome can be categorized into 2 binary levels, and if appropriate probability plot used to present the relationship. SPSS 20.0.0 and Minitab 17.1.0 software package used to make the statistical analysis, p-value considered when appropriate to be significant if $<0.05$.

\section{RESULTS}

\section{Characteristics of the whole group}

In the Table 1, we can see the characteristics of the whole study group; the mean age is 26.1 years with 3.8 years standard deviation (SD), the body mass is $26.2 \pm 3.0 \mathrm{~kg} / \mathrm{m}^{2}$. More than $70 \%$ of the women did not conceive before ( 57 out of 80 ) and more than $20 \%$ of the women have one or more abortions. The mean duration of infertility is 2.5 years.

\section{The two subgroups after day 7}

All the included women ( 80 women) were started on letrozole therapy, then transvaginal ultrasound was done at day 7 or 8 of the cycle, and after that, the women were sorted into two groups according to the size of the dominant follicle at that day, in the first group (Group A) with the size more than $10 \mathrm{~mm}$, while the other group (Group B) includes women with $<10 \mathrm{~mm}$ follicle. Table 2 shows the results of

Table 1: The characteristics of the whole group ( $n=80$ women)

\begin{tabular}{ll}
\hline Variables & Value \\
\hline Mean age in years \pm SD & $26.1 \pm 3.8$ \\
Mean BMI \pm SD & $26.2 \pm 3.0$ \\
Parity (number of women; \%) & \\
Null & $57 ; 71.2$ \\
1 & $17 ; 21.2$ \\
2 & $5 ; 6.2$ \\
3 & $1 ; 1.2$ \\
Abortion (number of women; \%) & $63 ; 78.8$ \\
0 & $13 ; 16.2$ \\
1 & $4 ; 5$ \\
2 & $2.5 \pm 1.1$ \\
\hline Mean duration of infertility (years) \pm SD &
\end{tabular}

Table 2: Comparison between the characteristics of the subgroup A (letrozole only) and subgroup B (letrozole and gonadotropin)

\begin{tabular}{llll}
\hline Variables & Subgroup A (n=48) & Subgroup B (n=32) & p value \\
\hline Mean age in years \pm SD & $24.4 \pm 2.0$ & $28.6 \pm 4.5$ & $<0.001$ \\
Mean BMI \pm SD & $24.5 \pm 1.9$ & $28.6 \pm 2.7$ & $<0.001$ \\
Parity (number of women; percent) & $39 ; 81.2$ & $19 ; 56.2$ & 0.089 \\
Null & $7 ; 14.6$ & $10 ; 31.2$ & $3 ; 9.4$ \\
1 & $2 ; 4.2$ & $1 ; 3.1$ & 0.747 \\
2 & $0 ; 0.0$ & $25 ; 78.1$ & \\
3 & & $6 ; 18.8$ & $<0.001$ \\
Abortion (number of women; \%) & $38 ; 79.2$ & $1 ; 3.1$ & \\
0 & $7 ; 14.6$ & $3.2 \pm 1.3$ & \\
1 & $3 ; 6.3$ & & \\
2 & $2.1 \pm 0.7$ & & \\
Mean duration of infertility (years) \pm SD & & & \\
\hline
\end{tabular}


the comparison between the characteristic of two groups, where the Group A contains 48 women, while Group B contains 32 women. There is a significant difference in the age, where the mean age is 28.6 years in Group B versus 24.4 years in Group A $(p<0.001)$. Furthermore, the body mass index (BMI) was higher in Group B $(\mathrm{p}<0.001)$. The parity status and previous history of abortion were not significantly different between the two groups; this is on the contrary with the duration of infertility where the Group B has a longer duration of infertility with a statistically significant difference.

\section{Outcome of treatment}

Table 3 shows the different levels of outcome of the whole group whether they continue on letrozole (Subgroup A), or they received gonadotropin in addition to letrozole (Subgroup B), the pregnancy occurred in 54 women $(67.5 \%)$, and the success of ovulation was $91.3 \%$, with cumulative mean number of cycles $2.3(\mathrm{SD} \pm 0.8)$. The mean endometrial thickness was $8.7 \mathrm{~mm}$, and we had three miscarriage out of 54 pregnancies.

In $70 \%$ of the women, they developed only one dominant follicle and $26 \%$ of women developed two follicles. We had three cases of mild OHSS, while we had two women with twin pregnancy.

\section{Comparison of the outcome between the two subgroups}

Women in Subgroup A developed one dominant follicle in $97.9 \%$, while more than $70 \%$ of women in Group B developed two dominant follicles $(p<0.001)$, the number of cycles, and endometrial thickness showed no significant difference between the two subgroups.

The success of ovulation was significantly higher in Subgroup A than B $(97.9 \%$ vs. $81.3 \%, p=0.015)$. Furthermore, we reported the higher percentages of pregnancy in Subgroup A than B (72.9\% vs. $59.4 \%)$, but it was statistically not significant $(\mathrm{p}=0.2)$.

Table 3: The different levels of the outcome of the whole group

\begin{tabular}{ll}
\hline Variable & Number of women; \% \\
\hline Number of the follicle (s) & \\
1 & $53 ; 70.7$ \\
2 & $20 ; 26.7$ \\
3 & $2 ; 2.7$ \\
Mean endometrial & $8.7 \pm 0.5$ \\
thickness (mm) \pm SD & \\
Mean size of follicles (mm) \pm SD & $19.1 \pm 1.4$ \\
Mean cumulative number of & $2.3 \pm 0.8$ \\
cycles \pm SD & \\
Pregnancy & $54 ; 67.5$ \\
Successful ovulation & $73 ; 91.3$ \\
Miscarriage & $3 ; 5.6(3$ out of 54 women $)$ \\
(percent per pregnant ladies) & \\
OHSS & $3 ; 3.8$ \\
\hline OHSS: Ovarian hyperstimulation syndrome, SD: Standard deviation
\end{tabular}

As mentioned above, we reported three cases of abortion, 2 in the letrozole group and one in the second group; this difference was statistically not significant.

Although the three reported cases of mild OHSS were seen in the Subgroup B, again the difference was statistically not significant (Table 4).

Lower cycle number independently and significantly predict clinical pregnancy, while BMI has the modest effect to predict clinical pregnancy, in which the lower the BMI, the better the outcome (BMI lower than 24.3 had the best outcome to predict clinical pregnancy), as illustrated in Table 5 and Fig. 1.

\section{DISCUSSION}

A normogonadotrophic anovulatory PCOS woman (WHO 2) shows high variation in ovarian response to ovulation induction. Treatment form in this group should become more individually tailored, and therefore, it seems reasonable to implicate new approaches to the conventional WHO 2 anovulation treatment protocol [15]

Franik et al. reviewed 26 RCTs (5560 women) using Cochrane database systemic review at 2014 on the use of aromatase inhibitors for subfertile women with polycystic ovary and end up with a conclusion that letrozole had a better pregnancy and live birth rate than CC and the complication of OHSS was rare [16].

Brown and Farquhar at 2016 did a review of 28 RCTs (3377 women) comparing clomiphene and other antiestrogens for ovulation induction in PCOS and went out with a conclusion that gonadotropin was more effective than clomiphene in the rate of live birth and ongoing pregnancy [17].

The use of letrozole in combination with gonadotropin had been assessed by several studies, especially in clomiphene-resistant cases. Xi et al. at 2015 did a prospective study on the use of letrozole-gonadotropin versus clomiphene-gonadotropins in clomiphene-resistant infertile women with PCOS and found out that letrozole-gonadotropin use significantly reduced the duration and total gonadotropin dose needed for stimulation and that the rate of monofollicular development was significantly higher in comparison, this, in turn, supports the concept that letrozole combined with gonadotropin reduces the risk of hyperstimulation [18].

Malhotra et al. did a prospective randomized trial on letrozole alone or letrozole gonadotropin combination as the first line for superovulation in women with unexplained infertility undergoing intrauterine insemination and went out in a conclusion that better number of follicles and improved ET result in higher pregnancy rate in letrozole-human menopausal gonadotropin (HMG) protocol in comparison to letrozole alone protocol and letrozole-HMG should be preferred over letrozole as the first line for superovulation in unexplained infertility [19].

Table 4: Comparison between the outcome of the subgroup A (letrozole only) and subgroup B (letrozole and gonadotropin)

\begin{tabular}{lll}
\hline Variable & \multicolumn{2}{l}{ Number of women; \% } \\
\cline { 2 - 3 } & Group A (48) & Group B (32) \\
\hline Number of the follicle (s) & & $6 ; 22.2 \%$ \\
1 & $47 ; 97.7 \%$ & $19 ; 70.4 \%$ \\
2 & $1 ; 2.1 \%$ & $2 ; 7.4 \%$ \\
3 & 0 & $8.8 \pm 0.5$ \\
Mean endometrial thickness (mm) \pm SD & $8.7 \pm 0.5$ & $19.6 \pm 1.5$ \\
Mean size of follicles (mm) \pm SD & $18.8 \pm 1.2$ & $2.1 \pm 0.9$ \\
Mean cumulative number of cycles \pm SD & $2.4 \pm 0.7$ & $19 ; 59.4 \%$ \\
Pregnancy & $35 ; 72.9 \%$ & $26 ; 81.3 \%$ \\
Successful ovulation & $47 ; 97.9 \%$ & $1 ; 5.3$ \\
Miscarriage (percent per pregnant ladies) & $2 ; 5.7 \%$ & 0.312 \\
OHSS & 0 & 0.009 \\
\hline
\end{tabular}

OHSS: Ovarian hyperstimulation syndrome, SD: Standard deviation 
Table 5: Multivariate analysis of the predictors of a successful pregnancy for all women

\begin{tabular}{lll}
\hline Variables & OR $(\mathbf{9 5 \%} \mathbf{~ C I})$ & p value \\
\hline Cycle number & $0.021(0.002-0.215)$ & 0.001 \\
BMI & $0.527(0.256-1.084)$ & 0.082 \\
Mean size of follicle & $1.524(0.822-2.825)$ & 0.181 \\
Age & $1.329(0.858-2.058)$ & 0.203 \\
Type of therapy & $1.376(0.003-547.188)$ & 0.917 \\
Parity & $3.639(0.423-31.338)$ & 0.240 \\
Abortion & $1.550(0.460-5.225)$ & 0.480 \\
Duration of infertility & $0.621(0.230-1.676)$ & 0.347 \\
Mean number of follicle & $0.224(0.010-4.845)$ & 0.340 \\
Endometrial thickness & $2.442(0.422-14.137)$ & 0.319 \\
Day in the cycle & $1.763(0.557-5.582)$ & 0.335 \\
Day of stimulation & $1.070(0.216-5.312)$ & 0.934 \\
\hline
\end{tabular}

$\mathrm{R}^{2}=0.442$ (Cox and Snell). OR: Odds ratio, CI: Confidence intervals, BMI: Body mass index

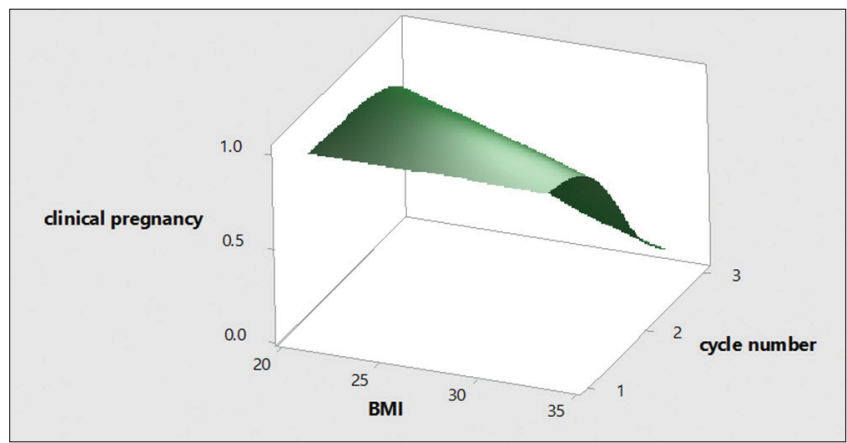

Fig. 1: Surface plot of the instant relationship between body mass index and cycle number to predict clinical pregnancy

Arya et al. at 2017 did retrospective cohort study on ovulation induction and $\mathrm{COH}$ using letrozole gonadotropin combination in PCOS and other fertility cases and end up in a conclusion of letrozole-low-dose gonadotropins combination appears to be effective across different causes of infertility for superovulation. The letrozole-low dose gonadotropin combination resulted in high rate of monofolliculogenesis, low occurrence of multiple gestations, and no case of OHSS or cycle cancellation [20].

Looking on all the studies together may justify adopting the use of letrozole as first-line ovulation induction in anovulatory infertility in PCOS and may possibly justify adopting the use of gonadotropin in combination with letrozole when the first one failed to achieve ovulation in the same cycle. In our study, the overall pregnancy rate was $67.5 \%(54 / 80)$, we reported the higher percentages of pregnancy in Subgroup A than B (72.9\% vs. $59.4 \%)$, but it was statistically not significant $(\mathrm{p}=0.2)$. The ovulation rate was $91.3 \%$ (73/90). The success of ovulation was significantly higher in Subgroup A than B (97.9\% vs. $81.3 \%, \mathrm{p}=0.015$ ). Depending on CD 7 ultrasound, the administration of low doses of exogenous FSH starting at that day can help to achive single dominant follicle selection in most of the women who fail to show a response to letrozole alone. This may explain the high rate of ovulation in both groups collectively. In those who did not show a response (8.7\%), the amount of exogenous FSH might have been too low to extend the window sufficiently for the selection of dominant follicles. In our study, number of follicle was monomolecular in $97.9 \%$ in Group A, while $26 \%$ of women developed 2 follicles in more than $70 \%$ in Group B $(\mathrm{p}<0.001)$. The number of cycles, endometrial thickness, miscarriage, twine, and OHSS showed no significant difference between the two subgroups. Looking into most of our study results, it goes with most of the results of the abovementioned literature [18-20].

By comparing demographic characters between Group A and Group B, we found significant differences regarding women age, BMI, and period of infertility. The use of prediction models may help to choose an individualized treatment pathway per PCOS woman in addition to CD7 ultrasound. In our ovulation induction protocol, we used fixed dose of letrozole (5 mg) only, not $2.5 \mathrm{mg}$ or $7.5 \mathrm{mg}$, protocol was supported by the retrospective study of Badawy et al. and randomized control trial of Polyzos et al. both showed that $5 \mathrm{mg}$ letrozole was more effective than other doses in the term of pregnancy rate [21,22]. Furthermore, in our protocol, we used $75 \mathrm{IU} \mathrm{rFSH}$, not $50 \mathrm{IU}$ or $100 \mathrm{IU}$, the dose that was supported by Streda et al. study at 2012 who showed that 50 IU daily is the appropriate starting dose to support ovulation, but the disadvantage may be an increased risk of cycle cancellation due to low ovarian response [23].

On the other hand, Akbari et al. use letrozole $5 \mathrm{mg}$ in combination with gonadotropin $100 \mathrm{IU}$ and compare it with clomiphene gonadotropin combination regime in unexplained infertility and they start adding gonadotropin on day 6-8 day of the menstrual cycle depending on that day response, and they went out in a conclusion that letrozole is a good alternative to CC, or it can be a first-line drug in ovarian stimulation and treatment of anovulation. Use of letrozole can induce ovulation comparable to CC without any adverse effect on endometrium and with comparable pregnancy rate and lower abortion rate compared with CC [24]. Based on Akbari et al. study, Pourali et al. at 2017 did a randomized trial on the same subject with the same study design and ended up with the same conclusion $[24,25]$. Malhotra et al. in their study also advocate using letrozole gonadotropin combination as a first-line treatment for unexplained infertility [19].

On the other hand, Kiran Chaudhary et al. at 2015 compare the efficacy of letrozole and low-dose gonadotropin combination with clomiphene and low-dose gonadotropin combination as a $\mathrm{COH}$ regime before intrauterine insemination in patients with unexplained infertility, and they found no significant differences in their primary and secondary outcome. In their study, they used $5 \mathrm{mg}$ letrozole with $150 \mathrm{IU}$ of gonadotropin but started at day 9 [26].

The last studies above [19, 24-26] advocating using letrozole with gonadotropin as a first-line treatment in unexplained infertility. Using letrozole alone or in combination with gonadotropin in the current study as a first-line treatment in PCOS may be reasonable since this approach may improve the success and minimize costs and risks, but it has a limitation regarding the sample size.

\section{CONCLUSION}

Letrozole alone or in combination with gonadotropin as a first-line treatment in PCOS may be reasonable since this approach may improve the success rate and minimize the overall costs and risks.

\section{ACKNOWLEDGMENTS}

This study was supported by the Department of Obstetrics and Gynecology in Al-Mustansiriyah Medical College and the Infertility Clinic in Al-Yarmouk Teaching Hospital/Baghdad/Iraq

\section{AUTHOR CONTRIBUTIONS}

Concept and collection of data, writing the article and critical review of the article, and final approval of the article - Fadia J Alizzi.

\section{CONFLICT OF INTEREST}

Fadia J Alizzi declares that she has no conflict of interest.

\section{REFERENCES}

1. Yildiz BO, Bozdag G, Yapici Z, Esinler I, Yarali H. Prevalence, phenotype and cardiometabolic risk of polycystic ovary syndrome under different diagnostic criteria. Hum Reprod 2012;27:3067-73.

2. Chang J, Azziz R, Legro R, Dewailly D, Franks S, Tarlatzis BC, et al. The Rotterdam ESHRE/ASRM-Sponsored PCOS Consensus Workshop Group. Revised 2003 Consensus on Diagnostic Criteria and Long-term Health Risks Related to Polycystic Ovary Syndrome[J] FertilSteril No. 00008111925 10.1016/j.fertnstert.2003.10.0042004; 
2004. p. 19-25.

3. Legro RS, Barnhart HX, Schlaff WD, Carr BR, Diamond MP, Carson SA, et al. Clomiphene, metformin, or both for infertility in the polycystic ovary syndrome. N Engl J Med 2007;356:551-66.

4. Kamphuis EI, Bhattacharya S, van der Veen F, Mol BW, Templeton A. Are we overusing IVF? BMJ 2014;348:g252.

5. Steimbach LM, Loução AD, Carraro CB, Tonin FS, Pontarolo R, Sanches AC. Metformin usage to induce ovulation in women with polycystic ovary syndrome: A meta-analysis. Int J Pharm PharmSci 2016;8:86-9

6. Thessaloniki ESHRE/ASRM-Sponsored PCOS Consensus Workshop Group. Consensus on infertility treatment related to polycystic ovary syndrome. Hum Reprod 2008;23:462-77.

7. Paul CB, Sudandiradoss C. Aromatase inhibitors-types and advantages. Int J Pharm PharmSci 2016;8:1-7.

8. Biljan MM, Hemmings R, Brassard N. The outcome of 150 babies following the treatment with letrozole or letrozole and gonadotropins. Fertil Steril 2005;84:S95.

9. Legro RS, Kunselman AR, Brzyski RG, Casson PR, Diamond MP, Schlaff WD, et al. The pregnancy in polycystic ovary syndrome II (PPCOS II) trial: Rationale and design of a double-blind randomized trial of clomiphene citrate and letrozole for the treatment of infertility in women with polycystic ovary syndrome. Contemp Clin Trials 2012;33:470-81

10. Herman A, Ron-El R, Golan A, Soffer Y, Bukovsky I, Caspi E. Overstimulated cycles under low-dose gonadotrophins in patients with polycystic ovary syndrome: Characterization and management. Hum Reprod 1993;8:30-4

11. Ghanem ME, Elboghdady LA, Hassan M, Helal AS, Gibreel A, Houssen $\mathrm{M}$, et al. Clomiphene citrate co-treatment with low dose urinary FSH versus urinary FSH for clomiphene-resistant PCOS: Randomized controlled trial. J Assist Reprod Genet 2013;30:1477-85.

12. Healey S, Tan SL, Tulandi T, Biljan MM. Effects of letrozole on superovulation with gonadotropins in women undergoing intrauterine insemination. Fertil Steril 2003;80:1325-9.

13. Mitwally MF, Casper RF. Aromatase inhibition reduces gonadotrophin dose required for controlled ovarian stimulation in women with unexplained infertility. Hum Reprod 2003;18:1588-97.

14. Sarhan A, Beydoun H, Jones HW Jr., Bocca S, Oehninger S, Stadtmauer L. Gonadotrophin ovulation induction and enhancement outcomes: Analysis of more than 1400 cycles. Reprod BioMed Online 2011;23:220-6.

15. Bedaiwy MA, Mousa NA, Esfandiari N, Forman R, Casper RF.
Follicular Phase dynamics with combined aromatase inhibitor and follicle stimulating hormone treatment. J Clin Endocrinol Metab 2007;92:825-33.

16. Franik S, Kremer JA, Nelen WL, Farquhar C. Aromatase inhibitors for subfertile women with polycystic ovary syndrome. Cochrane Database Syst Rev 2014;2014:Cd010287.

17. Brown J, Farquhar C. Clomiphene and other antioestrogens for ovulation induction in polycystic ovarian syndrome. Cochrane Database Syst Rev 2016;12:Cd002249.

18. Xi W, Liu S, Mao H, Yang Y, Xue X, Lu X. Use of letrozole and clomiphene citrate combined with gonadotropins in clomipheneresistant infertile women with polycystic ovary syndrome: A prospective study. Drug Des Dev Ther 2015;9:6001-8.

19. Malhotra N, Karmakar D, Kumar S. Letrozole alone or letrozole gonadotropin combination as the first line for superovulation in women with unexplained infertility undergoing intrauterine insemination (IUI): A prospective randomized trial. Fertil Steril 2012;98:S258.

20. Arya S, Kupesic-Plavsic S, Mulla ZD, Dwivedi AK, Crisp Z, Jose J, et al. Ovulation induction and controlled ovarian stimulation using letrozole gonadotropin combination: A single-centre retrospective cohort study. Eur J Obstetr Gynecol Reprod Biol 2017;218:123-8.

21. Badawy A, Abdel Aal I, Abulatta M. Clomiphene citrate or letrozole for ovulation induction in women with polycystic ovarian syndrome: A prospective randomized trial. Fertil Steril 2009;92:849-52.

22. Polyzos NP, Tzioras S, Badawy AM, Valachis A, Dritsas C, Mauri D. Aromatase inhibitors for female infertility: A systematic review of the literature. Reprod Biomed Online 2009;19:456-71.

23. Streda R, Mardesic T, Sobotka V, Koryntova D, Hybnerova L, Jindra M. Comparison of different starting gonadotropin doses (50, 75 and $100 \mathrm{IU}$ daily) for ovulation induction combined with intrauterine insemination. Arch Gynecol Obstetr 2012;286:1055-9.

24. Akbari S, Roozbahani MA, Roozbahani FA. Comparing of letrozole versus clomiphene citrate combined with gonadotropins in intrauterine insemination cycles. Iran J Reprod Med 2012;10:29-32.

25. Pourali L, Ayati S, Tavakolizadeh S, Soleimani H, Sani FT. Clomiphene citrate versus letrozole with gonadotropins in intrauterine insemination cycles: A randomized trial. Int J Reprod Biomed 2017;15:49-54.

26. Chaudhary K, Suri V, Dhaliwal L, Gainder S. Comparison of the efficacy of letrozole and low-dose gonadotropin combination with clomiphene and low-dose gonadotropin combination as a controlled ovarian stimulation regime prior to intrauterine insemination in patients with unexplained infertility. Fertil Sci Res 2014;1:98-103. 